\title{
Methadone plus contingency management or performance feedback reduces cocaine and opiate use in people with drug addiction
}

Schottenfeld RS, Chawarski MC, Pakes JR, et al. Methadone versus buprenorphine with contingency management or performance feedback for cocaine and opioid dependence. Am J Pyschiatry 2005; 162:340-8.

Does adding methadone to contingency management or performance feedback reduce illegal drug use in people with a history of drug addiction?

\section{METHODS}

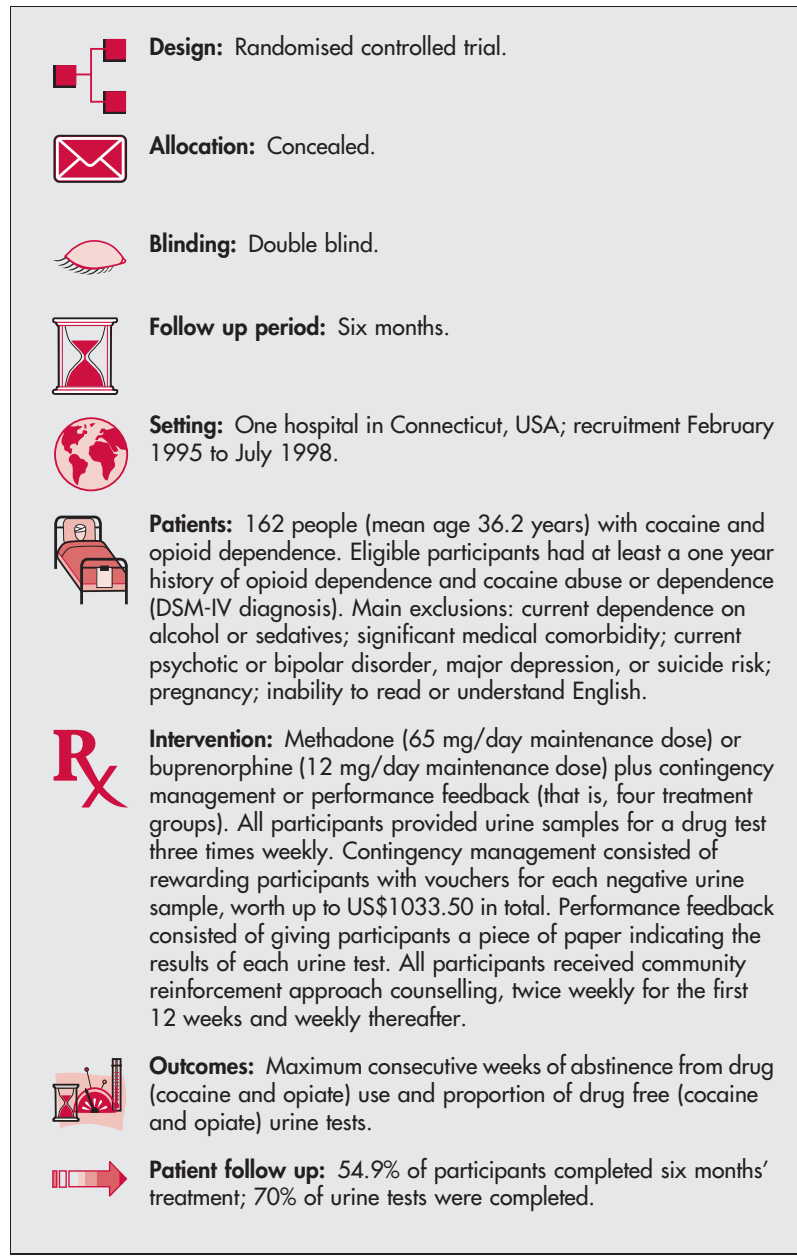

\section{MAIN RESULTS}

Adding methadone to either contingency management or performance feedback significantly increased abstinence from drug use and drug free urine tests at 24 weeks compared with adding buprenorphine (consecutive weeks of abstinence: 4.6 weeks with methadone $v$ 2.3 weeks with buprenorphine; $\mathrm{p}<0.05$; proportion of drug free urine tests: $36.3 \%$ with methadone $v 19.1 \%$ with buprenorphine; $\mathrm{p}<0.05$ ).

For correspondence: Richard Schottenfeld, The Department of Psychiatry, Yale University School of Medicine, 34 Park Street, Room S204, New Haven CT 06519, USA; Richard.schottenfeld@yale.edu

Sources of funding: The National Institute on Drug Abuse grants R01-DA09413, K24-DA-00445, T01-DA-13108, R01-DA-09803-04A2, and R01DA-012979.

\section{CONCLUSIONS}

Methadone plus contingency management or performance feedback increases abstinence from illegal drug use in people with a history of cocaine and opioid dependence.

\section{Commentary}

chottenfeld et al describe results of a trial evaluating independent and additive effects of medication and contingency management (CM) for reducing drug use. The study addresses two major issues in treating opioid dependence: (1) which medication is best; and (2) can the addition of CM improve outcomes?

The findings suggest that methadone engenders greater retention and more abstinence than buprenorphine. These data confirm earlier reports demonstrating increased efficacy of methadone relative to buprenorphine. ${ }^{1}$ The current study allowed for dose increases of both medications if continued use was occurring, a method commonly used in practice (which enhanced the external validity of the study). While buprenorphine has promise as a relatively safe maintenance medication with less overdose and abuse potential than methadone, results from this study suggest that patients may benefit more from the older medication.

This study also replicates other research findings on the efficacy of voucher based CM for decreasing substance use. ${ }^{2}$ While $C M$ enhances retention in non-opioid maintenance settings, this procedure rarely increases retention among methadone patients, presumably because methadone itself is a strong reinforcer. Some CM studies have failed to find beneficial effects of $\mathrm{CM}$ when abstinence from multiple substances is required to earn reinforcement, ${ }^{3}$ but Schottenfeld et al, along with one other study ${ }^{4}$ did demonstrate decreased use of both opioids and cocaine while contingent reinforcement was available. As in other studies, ${ }^{56}$ no synergistic effects of CM were noted when combined with another effective intervention.

The results from Schottenfeld et al suggest that methadone should be the initial therapy recommended for opioid dependent patients. CM may bolster some outcomes, but given its added expense, lower cost alternatives should be explored, ${ }^{4}$ in conjunction with large scale costbenefit analyses.

Nancy M Petry, PhD University of Connecticut Health Center, Farmington, CT, USA

1 Amato L, Davoli M, Perucci CA, et al. An overview of systematic reviews of the effectiveness of opiate maintenance therapies: available evidence to inform clinical practice and research. J Subs Abuse Treat 2005;28:321-9.

2 Silverman K, Higgins ST, Brooner RK, et al. Sustained cocaine abstinence in methadone maintenance patients through voucher-based reinforcement therapy. Arch Gen Psychiatry 1996;53:409-15.

3 Piotrowski NA, Tusel DJ, Sees KL, et al. Contingency contracting with monetary reinforcers for abstinence from multiple drugs in a methadone program. Exp Clin Psychopharmacol 1999;7:399-411.

4 Petry NM, Martin B. Low-cost contingency management for treating cocaine- and opioid-abusing methadone patients. J Consulting Clin Psychology 2002:70:398-405.

5 Epstein DH, Hawkins WE, Covi L, et al. Cognitive-behavioral therapy plus contingency management for cocaine use: Findings during treatment and across 12-month follow-up. Psychol Addictive Behav 2003;17:73-82.

6 Rawson RA, Huber A, McCann M, et al. A comparison of contingency management and cognitive-behavioral approaches during methadone maintenance treatment for cocaine dependence. Arch Gen Psychiatry 2002;59:817-24 